\title{
Niedbalski, Johanna: Die ganze Welt des Vergnügens. Berliner Vergnügungsparks der 1880er bis 1930er Jahre, 480 S., be.bra, Berlin 2018.
}

\author{
Habbo Knoch \\ Online publiziert: 29. Juli 2020 \\ (C) Der/die Autor(en) 2020
}

Als 1955 das erste „Disneyland“ in Kalifornien eröffnet wurde, war der moderne Vergnügungspark bereits seit mehr als einem Jahrhundert vor allem in Europa (zum Beispiel seit 1843 mit dem Kopenhagener „Tivoli“) und in den USA bekannt und hatte sich um 1900 fest etabliert. Seine Vorläufer reichten mit Sommergärten, Rummelplätzen und Welt-, Kolonial- oder Gewerbeausstellungen noch deutlich weiter zurück. Viele Vergnügungsparks der ,langen“ Jahrhundertwende um 1900 entstanden an bereits zuvor für ähnliche Zwecke genutzten Orten und verwendeten die bestehende Infrastruktur. Es hat auch mit dieser Geschichte sowie mit einer für diese Zeit der dynamischen Hochurbanisierung überaus typischen Fluidität von Formaten, Standorten und Unternehmern zu tun, dass es keine feste Definition ,des` Vergnügungsparks gibt. Stattdessen grenzt Johanna Niedbalski in ihrer Arbeit über Berliner Vergnügungsparks von den 1880er bis in die 1930er Jahre, die aus ihrer 2017 eingereichten Dissertation hervorgegangen ist, ihren Untersuchungsgegenstand von ähnlichen Unterhaltungsetablissements durch eine Verdichtung bestimmter Merkmale $\mathrm{ab}$, die sich an diesen Orten fand: In stadtnaher oder zentraler Lage vereinten sie ,,auf einem klar umgrenzten und meist eingezäunten Areal [...] weitgehend im Freien [...] in der Regel nur im Sommer [...] diverse zeitgenössische Freizeit-Angebote [...] wie Fahr-, Belustigungs- und Schaugeschäfte, Glücksspiele [...], technische Innovationen, Tanzveranstaltungen, Feste [...], Sportevents, Ausstellungen exotisch erscheinender Menschen, gehobene Ausflugsgastronomie und aufwändige Grünanlagen“ (S. 9f.). Eine möglichst große Vielfalt war das Programm; insofern verdichtete sich die von vielen Zeitgenossen für die als Funktionszentren expandierenden Innenstädte betonte Überreizung der Sinne in einem für die Besucher ausschließlich auf Unterhaltung ausgelegten Raum.

Diese ,ganze Welt des Vergnügens“ in ihrem einerseits großstädtischen, andererseits transnationalen Charakter für das Berlin der Jahrzehnte um 1900 präsentiert der vorliegende Band detailliert in zwei Teilen und auf einer breiten Quellenbasis sowohl von Archivquellen der Stadtverwaltung als auch von zeitgenössischen Presseberichten und Darstellungen. Zunächst wird jeweils die Geschichte der sechs

H. Knoch $(\bowtie)$

Universität zu Köln, Köln, Deutschland

E-Mail: habbo.knoch@uni-koeln.de 
bedeutendsten Berliner Parks chronologisch und im Kontext ähnlicher lokaler und internationaler Betriebe vorgestellt, die einen Zeitraum von 1880 bis 1939 umfasst. Einige dieser Parks bestanden nur für eine oder wenige Saisons (der Vergnügungspark der Berliner Gewerbeausstellung und der „Universum-Landes-AusstellungsPark“), die meisten für einige Jahre (die „Neue Welt“ in der Hasenheide, der ,Sternecker“ am Weißen See, das „Traumland“ in der Schönholzer Heide), und nur der „Luna-Park“, damals Europas größter Vergnügungspark, existierte weitgehend kontinuierlich von 1910 bis 1934, ohne aber in den 1920er Jahren an die Erfolge der Vorkriegszeit anknüpfen zu können.

Die Verfasserin arbeitet erstens den schnelllebigen Charakter der Vergnügungsparks hinsichtlich ihrer baulichen Anlage und der Veränderungen des Angebots heraus. Zweitens zeigt sie das ,,komplexe Wechselverhältnis lokaler Vorläufer und transnationaler Vorbilder“ (zum Beispiel von Coney Island in New York) auf (S. 201). Drittens setzten sich die Vergnügungsparks von anderen Erholungsorten dieser Jahre durch den zunehmenden Einsatz technischer Innovationen ab, die ebenfalls infolge transnationaler Transfers nach Berlin kamen. Dies trug zusammen mit einer stärker großunternehmerischen und ebenfalls transnationalen Organisation in den 1910er Jahren zu einer Angleichung bis Standardisierung des Angebots bei. Insgesamt bedeuteten aber Krieg, Inflation und Weltwirtschaftskrise massive Einschnitte für die auf eine hohe Besucherfrequenz und zahlungsfreudige Gäste der neuen Mittel- und Oberschichten angelegten Parks, die zum Niedergang dieses Unterhaltungstypus in Berlin - wie es sich zum Beispiel auch für Grandhotels feststellen lässt - seit den 1920er Jahren beigetragen haben.

Im zweiten Teil des Buches geht Niedbalski auf insgesamt acht Erlebnisdimensionen des „Vergnügens im Park“ näher ein: Tempo, Exotismus, Innovation, Spektakel, Sport, Erotik, Gedränge und Opulenz. Diese Dimensionen verdeutlicht sie jeweils an zwei bis drei Beispielen. So spielten Hochfahrgeschäfte für den Reiz der Geschwindigkeit eine zentrale Rolle, Inszenierungen von „Kairo“ und „Kongo" verdeutlichen den ungebrochenen Kolonialismus, technische Errungenschaften wie Rolltreppen wurden hier mit zum ersten Mal eingesetzt. Viele dieser Erlebnisdimensionen betreffen unmittelbare körperliche Aktivitäten wie das Reiten, Boxen oder Schwimmen, den Tanz oder das „Teufelsrad“, eine Plattform, bei der durch drehende Bewegungen die Fahrgäste gleichsam ineinander rutschten. Zwischen wie an den Attraktionen prägte den Besuch gerade in Hochphasen der dauernde Kontakt zu anderen Menschen, der sich bei konkreten Festveranstaltungen oder in den gastronomischen Bereichen noch einmal verstärkte. Gerahmt wurde der gesamte Park durch das merkliche Bemühen, sich durch opulente Angebote von einfacheren Amüsierbetrieben abzusetzen, die auch moralisch in größerem Verruf standen.

Die Darstellung der Erlebnisdimensionen mündet in der überzeugenden These, dass es nicht um ein alltagsabgewandtes, ,reines “ Vergnügen ging, sondern die Angebote und Praktiken vielmehr dann erfolgreich waren, wenn sie den Besuchern einen Bezug zu ihrem großstädtischen Leben vermittelten. Diese Verkopplung machte die Parks zu wichtigen Orten eines Erlernens von großstädtischen Verhaltensweisen, Körperlichkeiten, Fähigkeiten und Kenntnissen, die zur ,inneren Urbanisierung“ (Gottfried Korff) maßgeblich beitrugen. Dies geschah durch gerade entwickelte Innovationen oder den Fokus auf das Außergewöhnliche in einer besonders intensiven 
Atmosphäre animierter Sinnesreize, barg aber gerade durch diesen Akzent die Gefahr, zum „langweiligen Abklatsch bekannter Genres“ zu werden (S. 398). Auch in anderer Hinsicht waren die Vergnügungsparks ein Spiegel und Verstärker der modernen Großstadtgesellschaft: Grundsätzlich waren die Parks relativ offen für viele Schichten (und zunehmend auch für Frauen) und wurden auch weithin genutzt (jedoch waren Unterschichten und Jugendliche nicht erwünscht), aber die beträchtlichen sozialen Diskrepanzen blieben vor allem in der Zeit des Kaiserreichs aufgrund der durch die Preise gestaffelten Zugänglichkeit des Angebots erhalten.

Die Arbeit Niedbalskis kann überzeugend aufzeigen, dass die Vergnügungsparks wie Warenhäuser oder Grandhotels ein hybrides „Potpourri“ (S. 9) an Angeboten vorhielten und dabei viele Elemente anderer Typen von Unterhaltungsangeboten wie Zoos, Tanzlokale oder Varietés in sich aufnahmen. Kommerzialisierung und transnationale Transfer waren dabei eng miteinander verschränkt. Das lange Ende der meisten Vergnügungsparks der hier untersuchten Art hatte mit der Veränderung von (Groß-)Stadt und Urbanisierung zu tun. Räumliche Nähe und verkehrstechnische Verschränkung mit den Stadtzentren oder deren traditionellen Vergnügungsräumen waren im Zuge der wachsenden Mobilität und anderen funktionalen Nutzungen von Stadträumen - unter anderem durch Grünzonen als Erholungsräume, vor allem aber durch dichte Bebauungen und die automobile Infrastruktur - nicht mehr realisierbar und immer weniger gewünscht: Die Zukunft des Vergnügungsparks lag weitgehend in der Erschließung dezentraler Großareale, die zudem günstiger im Unterhalt und der Erreichbarkeit für Menschen waren und sind, die nicht in der Stadt selbst wohnen. Der ,Erfolg' der urbanen Vergnügungsparks lag mithin auch darin, zur Stabilisierung der innerstädtischen Urbanisierung beigetragen zu haben, sodass sie in der zweiten Hälfte des 20. Jahrhunderts zur Urbanisierung der Peripherie beitrugen.

Die vorliegende Monografie stellt eine aus historischer Perspektive für Berlin oder vergleichbare deutsche Großstädte bislang nicht geleistete Grundlagenarbeit dar, deren Aufbau, Gliederung und Argumentation überzeugen und schlüssig sind. Das Wechselspiel zwischen konkreten Beispielen und übergeordneten Interpretationen sowieso Systematisierungen ist gelungen. Aufgrund der Quellenzitate vor allem aus veröffentlichten Darstellungen gelingt der Verfasserin ein überaus anschaulicher Einblick in die Angebote der Vergnügungsparks. Die Interpretation hätte noch gewonnen, wenn diese Phänomenologie stärker mit Überlegungen zur Subjektmodellierung um 1900 einerseits, der Rolle der Medialisierung, Simulation und des ,Spektakels“ andererseits verbunden worden wäre. Die These des Beitrags zur ,inneren Urbanisierung“ ist überzeugend, allerdings wäre auch hier genauer nach abweichenden Verhaltensweisen zu fragen gewesen, die sich nicht in das oszillierende Gesamtbild von Park und Stadt gefügt haben: Das Vergnügen war nicht die ganze Welt dieser Parks.

Funding Open Access funding provided by Projekt DEAL.

Open Access Dieser Artikel wird unter der Creative Commons Namensnennung 4.0 International Lizenz veröffentlicht, welche die Nutzung, Vervielfältigung, Bearbeitung, Verbreitung und Wiedergabe in jeglichem Medium und Format erlaubt, sofern Sie den/die ursprünglichen Autor(en) und die Quelle ordnungsgemäß nennen, einen Link zur Creative Commons Lizenz beifügen und angeben, ob Änderungen vorgenommen wurden. 
Die in diesem Artikel enthaltenen Bilder und sonstiges Drittmaterial unterliegen ebenfalls der genannten Creative Commons Lizenz, sofern sich aus der Abbildungslegende nichts anderes ergibt. Sofern das betreffende Material nicht unter der genannten Creative Commons Lizenz steht und die betreffende Handlung nicht nach gesetzlichen Vorschriften erlaubt ist, ist für die oben aufgeführten Weiterverwendungen des Materials die Einwilligung des jeweiligen Rechteinhabers einzuholen.

Weitere Details zur Lizenz entnehmen Sie bitte der Lizenzinformation auf http://creativecommons.org/ licenses/by/4.0/deed.de.

\section{Schmied, Doris/Wüstenrot Stiftung (Hrsg.): Große Dörfer - Kleine Städte (RURAL, Bd. 9), 148 S., Cuvillier, Göttingen 2018.}

\section{Lina Schröder}

Online publiziert: 22. September 2020

(C) Der/die Autor(en) 2020

Das Fazit des vorliegenden Tagungsbands vom 20. Dorfsymposium (2016) spiegelt die erfolgreiche Arbeit des 1977 gegründeten interdisziplinären Arbeitskreises für Dorfentwicklung: Als Gründungsmotiv gibt Gerhard Henkel in seinem Rückblick eine Phase des tiefgreifenden Wandels des Dorfes an (S. 135). Zur effektiven Erarbeitung aktueller Probleme wurde sich damals bewusst auf eine interdisziplinäre und an der Gegenwart orientierte Herangehensweise verständigt (S. 140). Walter Christallers Zentralorttheorie wurde dabei kritisch hinterfragt, da diese zu einem planvollen Abbau von Autonomie, Infrastruktur und Arbeitsplätzen im Zuge der Herabqualifizierung deutscher Dörfer geführt habe (S. 146).

Die weiteren sieben verständlich geschriebenen Aufsätze stammen aus der Feder von elf Autoren, unter anderem aus der Politikwissenschaft, Geografie, Soziologie und Architektur. Jeder Beitrag beginnt mit einer kurzen Zusammenfassung sowohl in deutscher als auch in englischer Sprache und wird durch Abbildungen und Tabellen in den jeweiligen Aussagen unterstützt. An der jüngsten Vergangenheit orientiert, diskutieren sie unter anderem die Folgen der Schrumpfungsprozesse in der ländlichen Bevölkerung und betonen, dass große Dörfer und kleine Städte fächerübergreifend von der Forschung vernachlässigt wurden. Eine klare Definition bezüglich der

L. Schröder $(\bowtie)$

Julius-Maximilians-Universität Würzburg, Würzburg, Deutschland

E-Mail: lina.schroeder@uni-wuerzburg.de 